\title{
Cross-Intersecting Families of Vectors
}

\author{
János Pach ${ }^{1}$ and Gábor Tardos ${ }^{2}$ \\ 1 École Polytechnique Fédérale de Lausanne \\ pach@renyi.hu \\ 2 Rényi Institute of Mathematics, Budapest \\ and Center for Discrete Mathematics, Zhejiang Normal University \\ tardos@renyi.hu
}

\begin{abstract}
Given a sequence of positive integers $p=\left(p_{1}, \ldots, p_{n}\right)$, let $S_{p}$ denote the family of all sequences of positive integers $x=\left(x_{1}, \ldots, x_{n}\right)$ such that $x_{i} \leq p_{i}$ for all $i$. Two families of sequences (or vectors), $A, B \subseteq S_{p}$, are said to be $r$-cross-intersecting if no matter how we select $x \in A$ and $y \in B$, there are at least $r$ distinct indices $i$ such that $x_{i}=y_{i}$. We determine the maximum value of $|A| \cdot|B|$ over all pairs of $r$ cross-intersecting families and characterize the extremal pairs for $r \geq 1$, provided that $\min p_{i}>r+1$. The case $\min p_{i} \leq r+1$ is quite different. For this case, we have a conjecture, which we can verify under additional assumptions. Our results generalize and strengthen several previous results by Berge, Borg, Frankl, Füredi, Livingston, Moon, and Tokushige, and answers a question of Zhang.
\end{abstract}

\section{Introduction}

The Erdös-Ko-Rado theorem [7] states that for $n \geq 2 k$, every family of pairwise intersecting $k$-element subsets of an $n$-element set consists of at most $\left(\begin{array}{l}n-1 \\ k-1\end{array}\right)$ subsets, as many as the star-like family of all subsets containing a fixed element of the underlying set. This was the starting point of a whole new area within combinatorics: extremal set theory; see [12, 3], 6], 8]. The Erdös-Ko-Rado theorem has been extended and generalized to other structures: to multisets, divisors of an integer, subspaces of a vector space, families of permutations, etc. It was also generalized to "cross-intersecting" families, i.e., to families $A$ and $B$ with the property that every element of $A$ intersects all elements of $B$; see Hilton [13, Moon [16], and Pyber [17.

For any positive integer $k$, we write $[k]$ for the set $\{1, \ldots, k\}$. Given a sequence of positive integers $p=\left(p_{1}, \ldots, p_{n}\right)$, let

$$
S_{p}=\left[p_{1}\right] \times \cdots \times\left[p_{n}\right]=\left\{\left(x_{1}, \ldots, x_{n}\right): x_{i} \in\left[p_{i}\right] \text { for } i \in[n]\right\} .
$$

J. Pach is supported by OTKA under ERC projects GraDR and ComPoSe 10EuroGIGA-OP-003, and by Swiss National Science Foundation Grants 200020144531 and 200021-137574.

G. Tardos is supported by OTKA grant NN-102029, the "Lendület" project of the Hungarian Academy of Sciences and by EPFL. 
We will refer to the elements of $S_{p}$ as vectors. The Hamming distance between the vectors $x, y \in S_{p}$ is $\left|\left\{i \in[n]: x_{i} \neq y_{i}\right\}\right|$ and is denoted by $d(x, y)$. Let $r \geq 1$ be an integer. Two vectors $x, y \in S_{p}$ are said to be $r$-intersecting if $d(x, y) \leq n-r$. (This term originates in the observation that if we represent a vector $x=\left(x_{1}, \ldots, x_{n}\right) \in S_{p}$ by the set $\left\{\left(i, x_{i}\right): i \in[n]\right\}$, then $x$ and $y \in S_{p}$ are $r$-intersecting if and only if the sets representing them have at least $r$ common elements.) Two families $A, B \subseteq S_{p}$ are $r$-cross-intersecting, if every pair $x \in A, y \in B$ is $r$-intersecting. If $(A, A)$ is an $r$-cross-intersecting pair, we say $A$ is $r$-intersecting. We simply say intersecting or cross-intersecting to mean 1-intersecting or 1-cross-intersecting, respectively.

The investigation of the maximum value for $|A| \cdot|B|$ for cross-intersecting pairs of families $A, B \subseteq S_{p}$ was initiated by Moon [16]. She proved, using a clever induction argument, that in the special case when $p_{1}=p_{2}=\cdots=p_{n}=k$ for some $k \geq 3$, every cross-intersecting pair $A, B \subseteq S_{p}$ satisfies

$$
|A| \cdot|B| \leq k^{2 n-2},
$$

with equality if and only if $A=B=\left\{x \in S_{p}: x_{i}=j\right\}$, for some $i \in[n]$ and $j \in[k]$. In the case $A=B$, Moon's theorem had been discovered by Berge [2, Livingston [15], and Borg [4. See also Stanton [18. In his report on Livingston's paper, published in the Mathematical Reviews, Kleitman gave an extremely short proof for the case $A=B$, based on a shifting argument. Zhang [20] established a somewhat weaker result, using a generalization of Katona's circle method [14. Note that for $k=2$, we can take $A=B$ to be any family of $2^{n-1}$ vectors without containing a pair $\left(x_{1}, \ldots, x_{n}\right),\left(y_{1}, \ldots, y_{n}\right)$ with $x_{i}+y_{i}=3$ for every $i$. Then $A$ is an intersecting family with $|A|^{2}=2^{2 n-2}$, which is not of the type described in Moon's theorem.

Moon also considered $r$-cross-intersecting pairs in $S_{p}$ with $p_{1}=p_{2}=\cdots=$ $p_{n}=k$ for some $k>r+1$, and characterized all pairs for which $|A| \cdot|B|$ attains its maximum, that is, we have

$$
|A| \cdot|B|=k^{2(n-r)} .
$$

The assumption $k>r+1$ is necessary. See Tokushige [19], for a somewhat weaker result, using algebraic techniques.

Zhang 20] suggested that Moon's results may be extended to arbitrary sequences of positive integers $p=\left(p_{1}, \ldots, p_{n}\right)$. The aim of this note is twofold: (1) to establish such an extension under the assumption $\min _{i} p_{i}>r+1$, and (2) to formulate a conjecture that covers essentially all other interesting cases. We verify this conjecture in several special cases.

We start with the special case $r=1$, which has also been settled independently by Borg [5], using different techniques.

Theorem 1. Let $p=\left(p_{1}, \ldots, p_{n}\right)$ be a sequence positive integers and let $A, B \subseteq$ $S_{p}$ form a pair of cross-intersecting families of vectors.

We have $|A| \cdot|B| \leq\left|S_{p}\right|^{2} / k^{2}$, where $k=\min _{i} p_{i}$. Equality holds for the case $A=B=\left\{x \in S_{p}: x_{i}=j\right\}$, whenever $i \in[n]$ satisfies $p_{i}=k$ and $j \in[k]$. For $k \neq 2$, there are no other extremal cross-intersecting families. 
We say that a coordinate $i \in[n]$ is irrelevant for a set $A \subseteq S_{p}$ if, whenever two elements of $S_{p}$ differ only in coordinate $i$ and $A$ contains one of them, it also contains the other. Otherwise, we say that $i$ is relevant for $A$.

Note that no coordinate $i$ with $p_{i}=1$ can be relevant for any family. Each such coordinate forces an intersection between every pair of vectors. So, if we delete it, every $r$-cross-intersecting pair becomes $(r-1)$-cross-intersecting. Therefore, from now on we will always assume that we have $p_{i} \geq 2$ for every $i$.

We call a sequence of integers $p=\left(p_{1}, \ldots, p_{n}\right)$ a size vector if $p_{i} \geq 2$ for all $i$. The length of $p$ is $n$. We say that an $r$-cross-intersecting pair $A, B \subseteq S_{p}$ is maximal if it maximizes the value $|A| \cdot|B|$.

Using this notation and terminology, Theorem 1 can be rephrased as follows.

Theorem 1'. Let $p=\left(p_{1}, \ldots, p_{n}\right)$ be a sequence of integers with $k=\min _{i} p_{i}>2$.

For any maximal pair of cross-intersecting families, $A, B \subseteq S_{p}$, we have $A=B$, and there is a single coordinate which is relevant for $A$. The relevant coordinate $i$ must satisfy $p_{i}=k$.

See Section 5 for a complete characterization of maximal cross-intersecting pairs in the $k=2$ case. Here we mention that only the coordinates with $p_{i}=2$ can be relevant for them, but for certain pairs, all such coordinates are relevant simultaneously. For example, let $n$ be odd, $p=(2, \ldots, 2)$, and let $A=B$ consist of all vectors in $S_{p}$ which have at most $\lfloor n / 2\rfloor$ coordinates that are 1 . This makes $(A, B)$ a maximal cross-intersecting pair.

Let $T \subseteq[n]$ be a subset of the coordinates, let $x_{0} \in S_{p}$ be an arbitrary vector, and let $k$ be an integer satisfying $0 \leq k \leq|T|$. The Hamming ball of radius $k$ around $x_{0}$ in the coordinates $T$ is defined as the family

$$
B_{k}=\left\{x \in S_{p}:\left|\left\{i \in T: x_{i} \neq\left(x_{0}\right)_{i}\right\}\right| \leq k\right\} .
$$

Note that the pair $\left(B_{k}, B_{l}\right)$ is $(|T|-k-l)$-cross-intersecting. We use the word ball to refer to any Hamming ball without specifying its center, radius or its set of coordinates. A Hamming ball of radius 0 in coordinates $T$ is said to be obtained by fixing the coordinates in $T$.

For the proof of Theorem 1, we need the following statement, which will be established by induction on $n$, using the idea in [16].

Lemma 2. Let $1 \leq r<n$, let $p=\left(p_{1}, \ldots, p_{n}\right)$ be a size vector satisfying $3 \leq p_{1} \leq p_{2} \leq \cdots \leq p_{n}$ and let $A, B \subseteq S_{p}$ form a pair of $r$-cross-intersecting families. If

$$
\frac{2}{p_{r+1}}+\sum_{i=1}^{r} \frac{1}{p_{i}} \leq 1,
$$

then $|A| \cdot|B| \leq \prod_{i=r+1}^{n} p_{i}^{2}$. In case of equality, we have $A=B$ and this family can be obtained by fixing $r$ coordinates in $S_{p}$.

By fixing any $r$ coordinates, we obtain a "trivial" $r$-intersecting family $A=$ $B \subseteq S_{p}$. As was observed by Frankl and Füredi [9], not all maximal size $r$ intersecting families can be obtained in this way, for certain size vectors. They 
considered size vectors $p=(k, \ldots, k)$ with $n \geq r+2$ coordinates, and noticed that a Hamming ball of radius 1 in $r+2$ coordinates is $r$-intersecting. Moreover, for $k \leq r$, this family is strictly larger than the trivial $r$-intersecting family. See also [1].

On the other hand, as was mentioned before, for $k \geq r+2$, Moon [16] proved that among all $r$-intersecting families, the trivial ones are maximal.

This leaves open only the case $k=r+1$, where the trivial $r$-intersecting families and the radius 1 balls in $r+2$ coordinates have precisely the same size. We believe that in this case there are no larger $r$-intersecting families. For $r=1$, it can be and has been easily verified (and follows, for example, from our Theorem 1, which deals with the asymmetric case, when $A$ and $B$ do not necessarily coincide). Our Theorem 7 settles the problem also for $r>3$. The intermediate cases $r=2$ or 3 are still open, but they could possibly be handled by computer search.

Therefore, to characterize maximal size $r$-intersecting families $A$ or maximal $r$-cross-intersecting pairs of families $(A, B)$ for all size vectors, we cannot restrict ourselves to fixing $r$ coordinates. We make the following conjecture that can roughly be considered as a generalization of the Frankl-Füredi conjecture 9] that has been proved by Frankl and Tokushige [11. The generalization is twofold: we consider $r$-cross-intersecting pairs rather than $r$-intersecting families and we allow arbitrary size vectors not just vectors with all-equal coordinates.

Conjecture 3. If $1 \leq r \leq n$ and $p$ is a size vector of length $n$, then there exists a maximal pair of $r$-cross-intersecting families $A, B \subseteq S_{p}$, where $A$ and $B$ are balls. If we further have $p_{i} \geq 3$ for all $i \in[n]$, then all maximal pairs of $r$-cross-intersecting families consist of balls.

Note that the $r=1$ special case of Conjecture 3 is established by Theorem 1 . Some further special cases of the conjecture are settled in Theorem 7 .

It is not hard to narrow down the range of possibilities for maximal $r$-crossintersecting pairs that are formed by two balls, $A$ and $B$. In fact, the following theorem implies that all such pairs are determined up to isomorphism by the number of relevant coordinates. Assuming that Conjecture 3 is true, finding $\max |A| \cdot|B|$ for $r$-cross-intersecting families $A, B \subseteq S_{p}$ boils down to making numeric comparisons for pairs of balls obtained by various radii. In case $p_{i} \geq 3$ for all $i$ (and still assuming Conjecture 3), the same process also finds all maximal $r$-cross-intersecting pairs.

Theorem 4. Let $1 \leq r \leq n$ and let $p=\left(p_{1}, \ldots, p_{n}\right)$ be a size vector. If $A, B \subseteq$ $S_{p}$ form a maximal pair of $r$-cross-intersecting families, then either of them determines the other. In particular, $A$ and $B$ have the same set of relevant coordinates. Moreover, if $A$ is a ball of radius $l$ around $x_{0} \in S_{p}$ in a set of coordinates $T \subseteq[n]$, then $|T| \geq l+r$, and $B$ is a ball of radius $|T|-l-r$ around $x_{0}$ in the same set of the coordinates. Furthermore, we have $p_{i} \leq p_{j}$ for every $i \in T$ and $j \in[n] \backslash T$, and the radii of the balls differ by at most 1, that is, ||$T|-2 l-r| \leq 1$. 
Note that if $A=B$ for a maximal pair $(A, B)$ of $r$-cross-intersecting families, then $A$ is also a maximal size $r$-intersecting family. This is the case, in particular, if $A$ and $B$ are balls of equal radii. However, for many size vectors, no maximal $r$-cross-intersecting pair consists of $r$-intersecting families, as the maximal $r$ cross-intersecting pairs are often formed by balls whose radii differ by one. For example, for the size vector $p=(3,3,3,3,3)$, the largest 4 -intersecting family $C$ is obtained by fixing four coordinates, while the maximal 4-cross-intersecting pair is formed by a singleton $A=\{x\}$ and a ball $B$ of radius 1 around $x$ in all coordinates. Here we have $|A| \cdot|B|=11>|C|^{2}=9$.

As we have indicated above, we have been unable to prove Conjecture 3 in its full generality, but we were able to verify it in several interesting special cases. We will proceed in two steps. First we argue, using entropies, that the number of relevant coordinates in a maximal $r$-cross-intersecting family is bounded. Then we apply combinatorial methods to prove the conjecture under the assumption that the number of relevant coordinates is small.

In the case where there are many relevant coordinates for a pair of maximal $r$-cross-intersecting families, we use entropies to bound the size of the families and to prove

Theorem 5. Let $1 \leq r \leq n$, let $p=\left(p_{1}, \ldots, p_{n}\right)$ be a size vector, let $A, B \subseteq S_{p}$ form a maximal pair of $r$-cross-intersecting families, and let $T$ be the set of coordinates that are relevant for $A$ or $B$. Then neither the size of $A$ nor the size of $B$ can exceed

$$
\frac{\left|S_{p}\right|}{\prod_{i \in T}\left(p_{i}-1\right)^{1-2 / p_{i}}} .
$$

We use this theorem to bound the number of relevant coordinates $i$ with $p_{i}>2$. The number of relevant coordinates $i$ with $p_{i}=2$ can be unbounded; see Section 5 .

Theorem 6. Let $1 \leq r \leq n$, let $p=\left(p_{1}, \ldots, p_{n}\right)$ be a size vector, and let $A, B \subseteq S_{p}$ form a maximal pair of $r$-cross-intersecting families.

For the set of coordinates $T$ relevant for $A$ or $B$, we have

$$
\prod_{i=1}^{r} p_{i} \geq \prod_{i \in T}\left(p_{i}-1\right)^{1-2 / p_{i}},
$$

which implies that $\left|\left\{i \in T: p_{i}>2\right\}\right|<5 r$.

We can characterize the maximal $r$-cross-intersecting pairs for all size vectors $p$ satisfying $\min p_{i}>r+1$, and in many other cases.

Theorem 7. Let $1 \leq r \leq n$, let $p=\left(p_{1}, \ldots, p_{n}\right)$ be a size vector with $p_{1} \leq p_{2} \leq$ $\cdots \leq p_{n}$, and let $A, B \subseteq S_{p}$ form a pair of $r$-cross-intersecting families.

1. If $p_{1}>r+1$, we have $|A| \cdot|B| \leq \prod_{i=r+1}^{n} p_{i}^{2}$. In case of equality, $A=B$ holds and this family can be obtained by fixing $r$ coordinates in $S_{p}$. 
2. If $p_{1}=r+1>4$, we have $|A| \cdot|B| \leq \prod_{i=r+1}^{n} p_{i}^{2}$. In case of equality, $A=B$ holds and this family can be obtained either by fixing $r$ coordinates in $S_{p}$ or by taking a Hamming ball of radius 1 in $r+2$ coordinates $i$, all satisfying $p_{i}=r+1$.

3. There is a function $t(r)=r / 2+o(r)$ such that if $p_{1} \geq t(r)$ and $(A, B)$ is a maximal $r$-cross-intersecting pair, then the families $A$ and $B$ are balls in at most $r+3$ coordinates.

The proof of Theorem 7 relies on the following result.

Theorem 8. Let $1 \leq r \leq n$ and let $p$ be a size vector of length $n$.

1. If there exists a maximal pair of $r$-cross-intersecting families in $S_{P}$ with at most $r+2$ relevant coordinates, then there exists such a pair consisting of balls.

2. If $p_{i}>2$ for all $i \in[n]$ and $A, B \subseteq S_{p}$ form a maximal pair of $r$-crossintersecting families with at most $r+3$ relevant coordinates, then $A$ and $B$ are balls.

With an involved case analysis, it may be possible to extend Theorem 8 to pairs with more relevant coordinates. Any such an improvement would carry over to Theorem 7 .

All of our results remain meaningful in the symmetric case where $A=B$. For instance, in this case, Theorem 1 (also proved by Borg [5]) states that every intersecting family $A \subseteq S_{p}$ has at most $\left|S_{p}\right| / k$ members, where $k=\min _{i} p_{i}$. In case $k>2$, equality can be achieved only by fixing some coordinate $i$ with $p_{i}=k$. Note that in the case $A=B$ (i.e., $r$-intersecting families) the exact maximum size is known for size vectors $(q, \ldots, q)$, [1].

\section{Proofs of Theorems 4 and 1}

First, we verify Theorem 4 and a technical lemma (see Lemma 9 below) which generalizes the corresponding result in [16. Our proof is slightly simpler. Lemma 9 will enable us to deduce Lemma 2, the main ingredient of the proof of Theorem 1, presented at the end of the section.

Proof of Theorem 4. The first statement is self-evident: if $A, B \subseteq S_{p}$ form a maximal pair of $r$-cross-intersecting families, then

$$
B=\left\{x \in S_{p}: x \text { r-intersects } y \text { for all } y \in A\right\} .
$$

If a coordinate is irrelevant for $A$, then it is also irrelevant for $B$ defined by this formula. Therefore, by symmetry, $A$ and $B$ have the same set of relevant coordinates.

If $A$ is the Hamming ball around $x_{0}$ of radius $l$ in coordinates $T$, then we have $B=\emptyset$ if $|T|<l+r$, which is not possible for a maximal cross-intersecting 
family. If $|T| \geq l+r$, we obtain the ball claimed in the theorem. For every $i \in T$, $j \in[n] \backslash T$, consider the set $T^{\prime}=(T \backslash\{i\}) \cup\{j\}$ and the Hamming balls $A^{\prime}$ and $B^{\prime}$ of radii $l$ and $|T|-l-r$ around $x_{0}$ in the coordinates $T^{\prime}$. These balls form an $r$-cross-intersecting pair and in case $p_{i}>p_{j}$, we have $\left|A^{\prime}\right|>|A|$ and $\left|B^{\prime}\right|>|B|$, contradicting the maximality of the pair $(A, B)$.

Finally let $B_{l}$ be a ball of radius $l$ around some fixed vector $x$ in a fixed set $T$ of coordinates. We claim that the size $\left|B_{l}\right|$ of these balls is strictly log-concave, that is, we have

$$
\left|B_{l}\right|^{2}>\left|B_{l-1}\right| \cdot\left|B_{l+1}\right|
$$

for $1 \leq l<|T|$. As balls around different centers have the same size, we can represent the left-hand side as $\left|B_{l}\right|^{2}=|C|$, where

$$
C=\left\{(y, z) \mid y, z \in S_{p}, d(x, y) \leq l, d(y, z) \leq l\right\} .
$$

Similarly, the right-hand side can be represented as $\left|B_{l-1}\right| \cdot\left|B_{l+1}\right|=|D|$ with

$$
D=\left\{(y, z) \mid y, z \in S_{p}, d(x, y) \leq l-1, d(y, z) \leq l+1\right\} .
$$

We say that two pairs $(y, z)$ and $\left(y^{\prime}, z^{\prime}\right)$ (all four terms from $S_{p}$ ) are equivalent if $z=z^{\prime}$ and for every $i \in[n]$ we have either $y_{i}=y_{i}^{\prime}$ or $y_{i}, y_{i}^{\prime} \in\left\{x_{i}, z_{i}\right\}$. Let us fix an equivalence class $O$. For all $(y, z) \in O$, the element $z$ and some coordinates $y_{i}$ of $y$ are fixed. We call the remaining coordinates $i$ open. For an open coordinate $i$, the value of $y_{i}$ must be one of the non-equal values $x_{i}$ or $z_{i}$. If $m$ denotes the number of open coordinates in $O$, we have $|O|=2^{m}$. For a pair $(y, z) \in O$, the distance $d(x, y)=d_{1}+d_{2}(y)$, where $d_{1}$ is the number of fixed coordinates $i$ with $x_{i} \neq y_{i}$, while $d_{2}(y)$ is the number of open coordinates $i$ with $x_{i} \neq y_{i}$. Note that $d_{1}$ is constant for all elements of $O$, while $d_{2}(y)$ takes any value $j$ for $\left(\begin{array}{c}m \\ j\end{array}\right)$ members of $O$. Similarly, we can write $d(y, z)=d_{1}+\left(m-d_{2}(y)\right)$, as $y_{i} \neq z_{i}$ holds for the same fixed coordinates where $x_{i} \neq y_{i}$, and $y_{i}$ is equal to exactly one of $x_{i}$ and $z_{i}$ for an open coordinate $i$. Summarizing, we have

$$
\begin{gathered}
C \cap O=\left\{(y, z) \in O \mid d_{1}+m-l \leq d_{2}(y) \leq l-d_{1}\right\}, \\
D \cap O=\left\{(y, z) \in O \mid d_{1}+m-l-1 \leq d_{2}(y) \leq l-d_{1}-1\right\} .
\end{gathered}
$$

We claim that $|C \cap O| \geq|D \cap O|$. Indeed, if $l-d_{1}<m / 2$, then $C \cap O=D \cap O=\emptyset$. Otherwise, we have $|C \cap O|-|D \cap O|=\left(\begin{array}{c}m \\ l-d_{1}\end{array}\right)-\left(\begin{array}{c}m \\ l-d_{1}+1\end{array}\right) \geq 0$. Note also that equality holds only if $l-d_{1}<m / 2$ or $l-d_{1}>m$, in which cases $C$ and $D$ are disjoint from $O$ or contain $O$, respectively.

As $C$ contains at least as many pairs from every equivalence class as $D$ does, we have $|C| \geq|D|$. Equality cannot hold for all equivalence classes, so we have $|C|>|D|$, as claimed.

To finish the proof of the theorem, we need to verify that the pair $\left(B_{l_{1}}, B_{l_{2}}\right)$ is not maximal $r$-cross-intersecting if $r=|T|-l_{1}-l_{2}$ and $\left|l_{1}-l_{2}\right| \geq 2$. This follows from the log-concavity, because in case $l_{1} \geq l_{2}+2$ the pair $\left(B_{l_{1}-1}, B_{l_{2}+1}\right)$ is also $r$-cross-intersecting and $\left|B_{l_{1}-1}\right| \cdot\left|B_{l_{2}+1}\right|>\left|B_{l_{1}}\right| \cdot\left|B_{l_{2}}\right|$. 
The following lemma will also be used in the proof of Theorem 5, presented in the next section.

Lemma 9. Let $1 \leq r \leq n$, let $p=\left(p_{1}, \ldots, p_{n}\right)$ be a size vector, and let $A, B \subseteq S_{p}$ form a maximal pair of $r$-cross-intersecting families.

If $i \in[n]$ is a relevant coordinate for $A$ or $B$, then there exists a value $l \in\left[p_{i}\right]$ such that

$$
\begin{aligned}
& \left|\left\{x \in A: x_{i} \neq l\right\}\right| \leq|A| / p_{i}, \\
& \left|\left\{y \in B: y_{i} \neq l\right\}\right| \leq|B| / p_{i} .
\end{aligned}
$$

Proof. Let us fix $r, n, p, i, A$, and $B$ as in the lemma. By Theorem 4 , if a coordinate is irrelevant for $A$, then it is also irrelevant for $B$ and vice versa.

In the case $n=r$, we have $A=B$ and this family must be a singleton, so the lemma is trivially true. From now on, we assume that $n>r$ and hence the notion of $r$-cross-intersecting families is meaningful for $n-1$ coordinates.

Let $q=\left(p_{1}, \ldots, p_{i-1}, p_{i+1}, \ldots, p_{n}\right)$. For any $l \in\left[p_{i}\right]$, let

$$
\begin{aligned}
& A_{l}^{\prime}=\left\{x \in A: x_{i}=l\right\}, \\
& B_{l}^{\prime}=\left\{y \in B: y_{i}=l\right\},
\end{aligned}
$$

and let $A_{l}$ and $B_{l}$ stand for the families obtained from $A_{l}^{\prime}$ and $B_{l}^{\prime}$, respectively, by dropping their $i$ th coordinates. By definition, we have $A_{l}, B_{l} \subseteq S_{q}$, and $|A|=$ $\sum_{l}\left|A_{l}\right|$ and $|B|=\sum_{l}\left|B_{l}\right|$. Furthermore, for any two distinct elements $l, m \in\left[p_{i}\right]$, the families $A_{l}$ and $B_{m}$ are $r$-cross-intersecting, since the vectors in $A_{l}^{\prime}$ differ from the vectors in $B_{m}^{\prime}$ in the $i$ th coordinate, and therefore the $r$ indices where they agree must be elsewhere.

Let $Z$ denote the maximum product $\left|A^{*}\right| \cdot\left|B^{*}\right|$ of an $r$-cross-intersecting pair $A^{*}, B^{*} \subseteq S_{q}$. We have $\left|A_{l}\right| \cdot\left|B_{m}\right| \leq Z$ for all $l, m \in\left[p_{i}\right]$ with $l \neq m$. Adding an irrelevant $i$ th coordinate to the maximal $r$-cross-intersecting pair $A^{*}, B^{*} \subseteq S_{q}$, we obtain a pair $A^{* \prime}, B^{* \prime} \subseteq S_{p}$ with $\left|A^{* \prime}\right| \cdot\left|B^{* \prime}\right|=p_{i}^{2} Z$. Using the maximality of $A$ and $B$, we have $|A| \cdot|B| \geq p_{i}^{2} Z$. Let $l_{0}$ be chosen so as to maximize $\left|A_{l_{0}}\right| \cdot\left|B_{l_{0}}\right|$, and let $c=\left|A_{l_{0}}\right| \cdot\left|B_{l_{0}}\right| / Z$.

Assume first that $c \leq 1$. Then we have

$$
p_{i}^{2} Z \leq|A| \cdot|B|=\sum_{l, m \in\left[p_{i}\right]}\left|A_{l}\right| \cdot\left|B_{m}\right| \leq \sum_{l, m \in\left[p_{i}\right]} Z=p_{i}^{2} Z .
$$

Hence, we must have equality everywhere. This yields that $c=1$ and that $A_{l}$ and $B_{m}$ form a maximal $r$-cross-intersecting pair for all $l, m \in\left[p_{i}\right], l \neq m$. This also implies that $\left|A_{l}\right|=\left|A_{m}\right|$ for $l, m \in\left[p_{i}\right]$, from where the statement of the lemma follows, provided that $p_{i}=2$.

If $p_{i} \geq 3$, then all families $A_{l}$ must be equal to one another, since one member in a maximal $r$-cross-intersecting family determines the other, by Theorem 4 . This contradicts our assumption that the $i$ th coordinate was relevant for $A$.

Thus, we may assume that $c>1$. 
For $m \in\left[p_{i}\right], m \neq l_{0}$, we have $\left|A_{l_{0}}\right| \cdot\left|B_{m}\right| \leq Z=\left|A_{l_{0}}\right| \cdot\left|B_{l_{0}}\right| / c$. Thus,

$$
\left|B_{m}\right| \leq\left|B_{l_{0}}\right| / c
$$

which yields that $|B|=\sum_{m \in\left[p_{i}\right]}\left|B_{m}\right| \leq\left(1+\left(p_{i}-1\right) / c\right)\left|B_{l_{0}}\right|$. By symmetry, we also have

$$
\left|A_{m}\right| \leq\left|A_{l_{0}}\right| / c
$$

for $m \neq l_{0}$ and $|A| \leq\left(1+\left(p_{i}-1\right) / c\right)\left|A_{l_{0}}\right|$. Combining these inequalities, we obtain

$$
p_{i}^{2} Z \leq|A| \cdot|B| \leq\left(1+\left(p_{n}-1\right) / c\right)^{2}\left|A_{l_{0}}\right| \cdot\left|B_{l_{0}}\right|=\left(1+\left(p_{i}-1\right) / c\right)^{2} c Z .
$$

We solve the resulting inequality $p_{i}^{2} \leq c\left(1+\left(p_{i}-1\right) / c\right)^{2}$ for $c>1$ and conclude that $c \geq\left(p_{i}-1\right)^{2}$. This inequality, together with Equations (11) and (2), completes the proof of Lemma 9 .

Proof of Lemma 2. We proceed by induction on $n$.

Let $A$ and $B$ form a maximal $r$-cross-intersecting pair. It is sufficient to show that they have only $r$ relevant coordinates. Let us suppose that the set $T$ of their relevant coordinates satisfies $|T|>r$, and choose a subset $T^{\prime} \subseteq T$ with $\left|T^{\prime}\right|=r+1$. By Lemma 9, for every $i \in T^{\prime}$ there exists $l_{i} \in\left[p_{i}\right]$ such that the family $X_{i}=\left\{x \in B: x_{i} \neq l_{i}\right\}$ has cardinality $\left|X_{i}\right| \leq|B| / p_{i}$.

If we assume that

$$
\frac{2}{p_{r+1}}+\sum_{i=1}^{r} \frac{1}{p_{i}}<1
$$

holds (with strict inequality), then this bound of $\left|X_{i}\right|$ would suffice. In order to also be able to deal with the case

$$
\frac{2}{p_{r+1}}+\sum_{i=1}^{r} \frac{1}{p_{i}}=1
$$

we show that $\left|X_{i}\right|=|B| / p_{i}$ is not possible. Considering the proof of Lemma 9, equality here would mean that the families $A_{l}$ and $B_{l}$ (obtained by dropping the $i$ th coordinate from the vectors in the sets $\left\{x \in A: x_{i}=l\right\}$ and $\left\{y \in B: y_{i}=\right.$ $l\}$, respectively) satisfy the following condition: both $\left(A_{l_{i}}, B_{m}\right)$ and $\left(A_{m}, B_{l_{i}}\right)$ should be maximal $r$-cross-intersecting pairs for all $m \neq l_{i}$. By the induction hypothesis, this would imply that $A_{l_{i}}=B_{m}$ and $A_{m}=B_{l_{i}}$, contradicting that $\left|A_{m}\right|<\left|A_{l_{i}}\right|$ and $\left|B_{m}\right|<\left|B_{l_{i}}\right|$ (see (11), in view of $c>1$ ). Therefore, we have $\left|X_{i}\right|<|B| / p_{i}$.

Let $C=\left\{x \in S_{p} \quad: \quad x_{i}=1\right.$ for all $\left.i \in[r]\right\}$ be the $r$-intersecting family obtained by fixing $r$ coordinates in $S_{p}$. In the family $D=B \backslash\left(\bigcup_{i \in T^{\prime}} X_{i}\right)$, the coordinates in $T^{\prime}$ are fixed. Thus, we have

$$
|D| \leq \prod_{i \in[n] \backslash T^{\prime}} p_{i} \leq \prod_{i=r+2}^{n} p_{i}=|C| / p_{r+1}
$$


On the other hand, we have

$$
|D|=|B|-\sum_{i \in T^{\prime}}\left|X_{i}\right|>|B|\left(1-\sum_{i \in T^{\prime}} 1 / p_{i}\right) \geq|B|\left(1-\sum_{i=1}^{r+1} 1 / p_{i}\right) .
$$

Comparing the last two inequalities, we obtain

$$
|B|<\frac{|C|}{p_{r+1}\left(1-\sum_{i=1}^{r+1} 1 / p_{i}\right)} .
$$

By our assumption on $p$, the denominator is at least 1 , so that we have $|B|<|C|$. By symmetry, we also have $|A|<|C|$. Thus, $|A| \cdot|B|<|C|^{2}$ contradicting the maximality of the pair $(A, B)$. This completes the proof of Lemma 2.

Now we can quickly finish the proof of Theorem 1.

Proof of Theorem 1. Notice that Lemma 2 implies Theorem 1, whenever $k=$ $\min _{i} p_{i} \geq 3$. It remains to verify the statement for $k=1$ and $k=2$. For $k=1$, it follows from the fact that all pairs of vectors in $S_{p}$ are intersecting, thus the only maximal cross-intersecting pair is $A=B=S_{p}$.

Suppose next that $k=2$. For $x \in S_{p}$, let $x^{\prime} \in S_{p}$ be defined by $x_{i}^{\prime}=$ $\left(x_{i}+1 \bmod p_{i}\right)$ for $i \in[n]$. Note that $x \mapsto x^{\prime}$ is a permutation of $S_{p}$. Clearly, $x$ and $x^{\prime}$ are not intersecting, so we either have $x \notin A$ or $x^{\prime} \notin B$. As a consequence, we obtain that $|A|+|B| \leq\left|S_{p}\right|$, which, in turn, implies that $|A| \cdot|B| \leq\left|S_{p}\right|^{2} / 4$, as claimed. It also follows that all maximal pairs satisfy $|A|=|B|=\left|S_{p}\right| / 2$.

\section{Using entropy: Proofs of Theorems 5 and 6}

Proof of Theorem 5. Let $r, n, p, A, B$ and $T$ be as in the theorem. Let us write $y$ for a randomly and uniformly selected element of $B$. Lemma 9 implies that, for each $i \in T$, there exists a value $l_{i} \in\left[p_{i}\right]$ such that

$$
\operatorname{Pr}\left[y_{i}=l_{i}\right] \geq 1-1 / p_{i} .
$$

We bound the entropy $H\left(y_{i}\right)$ of $y_{i}$ from above by the entropy of the indicator variable of the event $y_{i}=l_{i}$ plus the contribution coming from the entropy of $y_{i}$ assuming $y_{i} \neq l_{i}$ :

$$
H\left(y_{i}\right) \leq h\left(1-1 / p_{i}\right)+\left(1 / p_{i}\right) \log \left(p_{i}-1\right)=\log p_{i}-\left(1-2 / p_{i}\right) \log \left(p_{i}-1\right),
$$

where $h(z)=-z \log z-(1-z) \log (1-z)$ is the entropy function, and we used that $1-1 / p_{i} \geq 1 / 2$.

For any $i \in[n] \backslash T$, we use the trivial estimate $H\left(y_{i}\right) \leq \log p_{i}$. By the subadditivity of the entropy, we obtain

$$
\log |B|=H(y) \leq \sum_{i \in[n]} H\left(y_{i}\right) \leq \sum_{i \in T}\left(\log p_{i}-\left(1-2 / p_{i}\right) \log \left(p_{i}-1\right)\right)+\sum_{i \in[n] \backslash T} \log p_{i},
$$


or, equivalently,

$$
|B| \leq \prod_{i \in T} \frac{p_{i}}{\left(p_{i}-1\right)^{1-2 / p_{i}}} \prod_{i \in[n] \backslash T} p_{i}=\frac{\left|S_{p}\right|}{\prod_{i \in T}\left(p_{i}-1\right)^{1-2 / p_{i}}}
$$

as required. The bound on $|A|$ follows by symmetry and completes the proof of the theorem.

Theorem 6 is a simple corollary of Theorem 5 .

Proof of Theorem 6 . Fixing the first $r$ coordinates, we obtain the family

$$
C=\left\{x \in S_{p}: x_{i}=1 \text { for all } i \in[r]\right\} .
$$

This family is $r$-intersecting. Thus, by the maximality of the pair $(A, B)$, we have

$$
|A| \cdot|B| \geq|C|^{2}=\left(\prod_{i=r+1}^{n} p_{i}\right)^{2} .
$$

Comparing this with our upper bounds on $|A|$ and $|B|$, we obtain the inequality claimed in the theorem.

To prove the required bound on the number of relevant coordinates $i$ with $p_{i} \neq 2$, we assume that the coordinates are ordered, that is, $p_{1} \leq p_{2} \leq \cdots \leq$

$p_{n}$. Applying the above estimate on $\prod_{i \in[r]} p_{i}$ and using $\left(p_{i}-1\right)^{1-2 / p_{i}}>p_{i}^{1 / 5}$ whenever $p_{i} \geq 3$, the theorem follows.

\section{Monotone families: Proofs of Theorems 8 and 7}

Given a vector $x \in S_{p}$, the set $\operatorname{supp}(x)=\left\{i \in[n]: x_{i}>1\right\}$ is called the support of $x$. A family $A \subseteq S_{p}$ is said to be monotone, if for any $x \in A$ and $y \in S_{p}$ satisfying $\operatorname{supp}(y) \subseteq \operatorname{supp}(x)$, we have $y \in A$.

For a family $A \subseteq S_{p}$, let us define its support as $\operatorname{supp}(A)=\{\operatorname{supp}(x): x \in$ $A\}$. For a monotone family $A$, its support is clearly subset-closed and it uniquely determines $A$, as $A=\left\{x \in S_{p}: \operatorname{supp}(x) \in \operatorname{supp}(A)\right\}$.

The next result shows that if we want to prove Conjecture 3 , it is sufficient to prove it for monotone families. This will enable us to establish Theorems 8 and 7 , that is, to verify the conjecture for maximal $r$-cross-intersecting pairs with a limited number of relevant coordinates. Note that similar reduction to monotone families appears also in [9].

Lemma 10. Let $1 \leq r \leq n$ and let $p$ be a size vector of length $n$.

There exists a maximal pair of $r$-cross-intersecting families $A, B \subseteq S_{p}$ such that both $A$ and $B$ are monotone.

If $p_{i} \geq 3$ for all $i \in[n]$, and $A, B \subseteq S_{p}$ are maximal $r$-cross-intersecting families that are not balls, then there exists a pair of maximal $r$-cross-intersecting families that consists of monotone families that are not balls and have no more relevant coordinates than $A$ or $B$. 
Proof. Consider the following shift operations. For any $i \in[n]$ and $j \in\left[p_{i}\right] \backslash\{1\}$, for any family $A \subseteq S_{p}$ and any element $x \in A$, we define

$$
\begin{aligned}
\phi_{i}(x) & =\left(x_{1}, \ldots, x_{i-1}, 1, x_{i+1}, \ldots, x_{n}\right), \\
\phi_{i, j}(x, A) & = \begin{cases}\phi_{i}(x) & \text { if } x_{i}=j \text { and } \phi_{i}(x) \notin A \\
x & \text { otherwise },\end{cases} \\
\phi_{i, j}(A) & =\left\{\phi_{i, j}(x, A): x \in A\right\} .
\end{aligned}
$$

Clearly, we have $\left|\phi_{i, j}(A)\right|=|A|$ for any family $A \subseteq S_{p}$. We claim that for any pair of $r$-cross-intersecting families $A, B \subseteq S_{p}$, the families $\phi_{i, j}(A)$ and $\phi_{i, j}(B)$ are also $r$-cross-intersecting. Indeed, if $x \in A$ and $y \in B$ are $r$-intersecting vectors, then $\phi_{i, j}(x, A)$ and $\phi_{i, j}(y, B)$ are also $r$-intersecting, unless $x$ and $y$ have exactly $r$ common coordinates, one of them is $x_{i}=y_{i}=j$, and this common coordinate gets ruined as $\phi_{i, j}(x, A)=x$ and $\phi_{i, j}(y, B)=\phi_{i}(y)$ (or vice versa). However, this is impossible, because this would imply that the vector $\phi_{i}(x)$ belongs to $A$, in spite of the fact that $\phi_{i}(x)$ and $y \in B$ are not $r$-intersecting.

If $(A, B)$ is a maximal $r$-cross-intersecting pair, then so is $\left(\phi_{i, j}(A), \phi_{i, j}(B)\right)$. When applying one of these shift operations changes either of the families $A$ or $B$, then the total sum of all coordinates of all elements decreases. Therefore, after shifting a finite number of times we arrive at a maximal pair of $r$-intersecting families that cannot be changed by further shifting. We claim that this pair $(A, B)$ is monotone. Let $y \in B$ and $y^{\prime} \in S_{p} \backslash B$ be arbitrary. We show that $B$ is monotone by showing that $\operatorname{supp}\left(y^{\prime}\right)$ is not contained in $\operatorname{supp}(y)$. Indeed, by the maximality of the pair $(A, B)$ and using the fact that $y^{\prime} \notin B$, there must exist $x^{\prime} \in A$ such that $x^{\prime}$ and $y^{\prime}$ are not $r$-cross-intersecting, and hence $\left|\operatorname{supp}\left(x^{\prime}\right) \cup \operatorname{supp}\left(y^{\prime}\right)\right|>n-r$. Applying "projections" $\phi_{i}$ to $x^{\prime}$ in the coordinates $i \in \operatorname{supp}\left(x^{\prime}\right) \cap \operatorname{supp}(y)$, we obtain $x$ with $\operatorname{supp}(x)=\operatorname{supp}\left(x^{\prime}\right) \backslash \operatorname{supp}(y)$. The shift operations $\phi_{i, j}$ do not change the family $A$, thus $A$ must be closed for the projections $\phi_{i}$ and we have $x \in A$. The supports of $x$ and $y$ are disjoint. Thus, their Hamming distance is $|\operatorname{supp}(x) \cup \operatorname{supp}(y)|$, which is at most $n-r$, as they are $r$-intersecting. Therefore, $\operatorname{supp}(x) \cup \operatorname{supp}(y)=\operatorname{supp}\left(x^{\prime}\right) \cup \operatorname{supp}(y)$ is smaller than $\operatorname{supp}\left(x^{\prime}\right) \cup \operatorname{supp}\left(y^{\prime}\right)$, showing that $\operatorname{supp}\left(y^{\prime}\right) \nsubseteq \operatorname{supp}(y)$. This proves that $B$ is monotone. By symmetry, $A$ is also monotone, which proves the first claim of the lemma.

To prove the second claim, assume that $p_{i} \geq 3$ for all $i \in[n]$. Note that Theorem 1 establishes the lemma in the case $r=1$, so from now on we can assume without loss of generality that $r \geq 2$. Let $A, B \subseteq S_{p}$ form a maximal $r$ cross-intersecting pair. By the previous paragraph, this pair can be transformed into a monotone pair by repeated applications of the shift operations $\phi_{i, j}$. Clearly, these operations do not introduce new relevant coordinates. It remains to check that the shifting operations do not produce balls from non-balls, that is, if $A, B \subseteq$ $S_{p}$ are maximal $r$-cross-intersecting families, and $A^{\prime}=\phi_{i, j}(A)$ and $B^{\prime}=\phi_{i, j}(B)$ are balls, then so are $A$ and $B$. In fact, by Theorem 4 it is sufficient to prove that one of them is a ball. 
We saw that $A^{\prime}$ and $B^{\prime}$ must also form a maximal $r$-cross-intersecting pair. Thus, by Theorem 4 , there is a set of coordinates $T \subseteq[n]$, a vector $x_{0} \in S_{p}$, and radii $l$ and $m$ satisfying $|T|=r+l+m$ and that $A^{\prime}$ and $B^{\prime}$ are the Hamming balls of radius $l$ and $m$ in coordinates $T$ around the vector $x_{0}$. We can assume that $i \in T$, because otherwise $A=A^{\prime}$ and we are done. We also have that $\left(x_{0}\right)_{i}=1$, as otherwise $A^{\prime}=\phi_{i, j}(A)$ is impossible. The vectors $x \in S_{p}$ such that $x_{i}=j$ and

$$
\left|\left\{k \in T: x_{k} \neq\left(x_{0}\right)_{k}\right\}\right|=l+1
$$

are called $A$-critical. Analogously, the vectors $y \in S_{p}$ such that $y_{i}=j$ and

$$
\left|\left\{k \in T: y_{k} \neq\left(x_{0}\right)_{k}\right\}\right|=m+1
$$

are said to be $B$-critical. By the definition of $\phi_{i, j}$, the family $A$ differs from $A^{\prime}$ by including some $A$-critical vectors $x$ and losing the corresponding vectors $\phi_{i}(x)$. Symmetrically, $B \backslash B^{\prime}$ consists of some $B$-critical vectors $y$ and $B^{\prime} \backslash B$ consists of the corresponding vectors $\phi_{i}(y)$. Let us consider the bipartite graph $G$ whose vertices on one side are the $A$-critical vectors $x$, the vertices on the other side are the $B$-critical vectors $y$ (considered as disjoint families, even if $l=m$ ), and $x$ is adjacent to $y$ if and only if $\left|\left\{k \in[n]: x_{k}=y_{k}\right\}\right|=r$. If $x$ and $y$ are adjacent, then neither the pair $\left(x, \phi_{i}(y)\right)$, nor the pair $\left(\phi_{i}(x), y\right)$ is $r$-intersecting. As $A$ and $B$ are $r$-cross-intersecting, for any pair of adjacent vertices $x$ and $y$ of $G$, we have $x \in A$ if and only if $y \in B$.

The crucial observation is that the graph $G$ is connected. Note that this is not the case if $p_{k}=2$ for some index $k \notin T$, since all $A$-critical vectors $x$ in a connected component of $G$ would have the same value $x_{k}$. However, we assumed that $p_{k}>2$ for $l \in[n]$. In this case, the $A$-critical vectors $x$ and $x^{\prime}$ have a common $B$-critical neighbor (and, therefore, their distance in $G$ is 2 ) if and only if the symmetric difference of the $l$ element sets $\left\{k \in T \backslash\{i\}: x_{k} \neq\left(x_{0}\right)_{k}\right\}$ and $\left\{k \in T \backslash\{i\}: x_{k}^{\prime} \neq\left(x_{0}\right)_{k}\right\}$ have at most $2 r-2$ elements. We assumed that $r>1$, so this means that all $A$-critical vectors are indeed in the same component of the graph $G$. Therefore, either all $A$-critical vectors belong to $A$ or none of them does. In the latter case, we have $A=A^{\prime}$. In the former case, $A$ is the Hamming ball of radius $l$ in coordinates $T$ around the vector $x_{0}^{\prime}$, where $x_{0}^{\prime}$ agrees with $x_{0}$ in all coordinates but in $\left(x_{0}^{\prime}\right)_{i}=j$. In either case, $A$ is a ball as required.

Proof of Theorem 8. By Lemma 10, it is enough to restrict our attention to monotone families $A$ and $B$. We may also assume that all coordinates are relevant (simply drop the irrelevant coordinates). Thus, we have $n \leq r+3$.

Denote by $U_{l}$ the Hamming ball of radius $l$ around the all- 1 vector in the entire set of coordinates $[n]$. Notice that the monotone families $A$ and $B$ are $r$-cross-intersecting if and only if for $a \in \operatorname{supp}(A)$ and $b \in \operatorname{supp}(B)$ we have $|a \cup b| \leq n-r$. We consider all possible values of $n-r$, separately.

If $n=r$, both families $A$ and $B$ must coincide with the singleton $U_{0}$.

If $n=r+1$, it is still true that either $A$ or $B$ is $U_{0}$, and hence both families are balls. Otherwise, both $\operatorname{supp}(A)$ and $\operatorname{supp}(B)$ have to contain at least one non-empty set, but the union of these sets has size at most $n-r=1$, so we have 
$\operatorname{supp}(A)=\operatorname{supp}(B)=\{\emptyset,\{i\}\}$ for some $i \in[n]$. This contradicts our assumption that the coordinate $i$ is relevant for $A$.

If $n=r+2$, we are done if $A=B=U_{1}$. Otherwise, we must have a 2-element set either in $\operatorname{supp}(A)$ or in $\operatorname{supp}(B)$. Let us assume that a 2-element set $\{i, j\}$ belongs to $\operatorname{supp}(A)$. Then each set $b \in \operatorname{supp}(B)$ must satisfy $b \subseteq\{i, j\}$. This leaves five possibilities for a non-empty monotone family $B$, as $\operatorname{supp}(B)$ must be one of the following set systems:

1. $\{\emptyset\}$,

2. $\{\emptyset,\{i\}\}$,

3. $\{\emptyset,\{j\}\}$,

4. $\{\emptyset,\{i\},\{j\}\}$, and

5. $\{\emptyset,\{i\},\{j\},\{i, j\}\}$.

Cases 2, 3, and 5 are not possible, because either $i$ or $j$ would not be relevant for $B$.

In case $1, A$ and $B$ are balls, as claimed. Nevertheless, this case is impossible as the radii of $A$ and $B$ differ by 2, contradicting Theorem 4 .

It remains to deal with case 4. Here $\operatorname{supp}(A)$ consists of the sets of size at most 1 and the 2 -element set $\{i, j\}$. Define

$$
C=\left\{x \in S_{p}: x_{k}=1 \text { for all } k \in[n] \backslash\{i, j\}\right\} .
$$

Note that $|A|+|B|=\left|U_{1}\right|+|C|$, because each vector in $S_{p}$ appears in the same number of sets on both sides. Thus, we have either $|A|+|B| \leq 2\left|U_{1}\right|$ or $|A|+|B| \leq 2|C|$. Since $|A|>|B|$, the above inequalities imply $|A| \cdot|B|<\left|U_{1}\right|^{2}$ or $|A| \cdot|B|<|C|^{2}$. This contradicts the maximality of the pair $(A, B)$, because both $U_{1}$ and $C$ are $r$-intersecting. The contradiction completes the proof of the case $n-r=2$.

To complete the proof of Theorem 8 , we need to deal with the case $n-r=3$, i.e., when there are $r+3$ relevant coordinates. Note that, as part 1 of Theorem 8 does not apply to this case, we have $p_{i} \geq 3$ for $i \in[n]$. This slightly simplifies the following case analysis, where we consider all containment-maximal pairs of families $(\operatorname{supp}(A), \operatorname{supp}(B))$ with the required condition on the size of the pairwise unions.

Before considering the individual cases, we make a few simple observations. First, we have

$$
\begin{aligned}
& \operatorname{supp}(A)=\{T \subseteq[n]|\forall U \in \operatorname{supp}(B):| T \cup U \leq 3\}, \\
& \operatorname{supp}(B)=\{U \subseteq[n]|\forall T \in \operatorname{supp}(A):| T \cup U \mid \leq 3 .
\end{aligned}
$$

These are non-empty sets and they determine the monotone sets $A$ and $B$.

We say that $i$ dominates $j$ in a set system $C$, if whenever $j \in T$ but $i \notin T$ for a set $T \in C$, then we have $(T \backslash\{j\}) \cup\{i\} \in C$. We say that $i$ is equivalent to $j$ in $C$ if $i$ dominates $j$ in $C$ and $j$ also dominates $i$. If $i$ dominates $j$ but $j$ does not dominate $i$, then we say that $i$ strictly dominates $j$. 
Note that if one of the statements " $i$ dominates $j$," " $i$ is equivalent to $j$," or " $i$ strictly dominates $j$ " holds in either $\operatorname{supp}(A)$ or $\operatorname{supp}(B)$, then the same statement holds in both families. If $i$ strictly dominates $j$ in $\operatorname{supp}(A)$, then we have $p_{i} \geq p_{j}$. Indeed, otherwise we would have $\left|A^{\prime}\right|>|A|$ and $\left|B^{\prime}\right|>|B|$ for the monotone families $A^{\prime}$ and $B^{\prime}$ whose supports $\operatorname{supp}\left(A^{\prime}\right)$, resp. $\operatorname{supp}\left(B^{\prime}\right)$, are obtained from $\operatorname{supp}(A), \operatorname{resp} . \operatorname{supp}(B)$, by switching the roles of $i$ and $j$. Since $A^{\prime}$ and $B^{\prime}$ are $r$-cross-intersecting, this contradicts the maximality of $(A, B)$.

For equivalent coordinates $i$ and $j$ in $\operatorname{supp}(A)$, we may assume by symmetry that $p_{i} \geq p_{j}$.

Case 1. First assume that $\operatorname{supp}(A)$ contains a 3 -element set $\{i, j, k\}$. Then all sets in $\operatorname{supp}(B)$ are contained in $\{i, j, k\}$. Therefore, $\operatorname{supp}(B)$ must be one of the following sets, up to a suitable permutation of the indices $i, j$, and $k$.

1. $\{\emptyset\}$,

2. $\{\emptyset,\{i\}\}$,

3. $\{\emptyset,\{i\},\{j\}\}$,

4. $\{\emptyset,\{i\},\{j\},\{k\}\}$,

5. $\{\emptyset,\{i\},\{j\},\{i, j\}\}$

6. $\{\emptyset,\{i\},\{j\},\{k\},\{i, j\}\}$,

7. $\{\emptyset,\{i\},\{j\},\{k\},\{i, j\},\{i, k\}\}$,

8. $\{\emptyset,\{i\},\{j\},\{k\},\{i, j\},\{i, k\},\{j, k\}\}$,

9. $\{\emptyset,\{i\},\{j\},\{k\},\{i, j\},\{i, k\},\{j, k\},\{i, j, k\}\}$.

In all of these families, $i$ dominates $j$ and $j$ dominates $k$. So we may assume $p_{i} \geq p_{j} \geq p_{k} \geq 3$.

Subcases $2,5,7$, and 9 are not possible, because $i$ is not a relevant coordinate for $A$ in them.

In subcase $1, A$ and $B$ are balls, but, as before, this case is still impossible, because the radii of $A$ and $B$ differ by 3 .

In subcase 3 , we apply Lemma 9 to the set $B$ and coordinate $i$ to obtain $\left(p_{i}-1\right)^{2} \leq p_{j}$, a contradiction.

In subcase 4 , a similar application of Lemma 9 yields $\left(p_{i}-1\right)^{2} \leq p_{j}+p_{k}-1$ with the only solution $p_{i}=p_{j}=p_{k}=3$. We have $\operatorname{supp}(A)=\operatorname{supp}\left(U_{2}\right) \cup$ $\{\{i, j, k\}\}$ and thus $|A|=\left|U_{2}\right|+8$. We further have $|B|=7$. We must have $n \geq 4$, so that $\left|U_{1}\right| \geq 9$ and $\left|U_{2}\right| \geq 33$. Using these estimates, we obtain $|A| \cdot|B|<$ $\left|U_{1}\right| \cdot\left|U_{2}\right|$, a contradiction.

In subcase 6 , we again start with Lemma 9 . It yields that $\left(p_{i}-1\right)^{2} p_{j} \leq$ $p_{j}+p_{k}-1$, a contradiction.

Finally, in subcase 8 , we have $\left(p_{i}-1\right)^{2}\left(p_{j}+p_{k}-1\right) \leq p_{j} p_{k}$ from Lemma 9 , a contradiction.

Case 2. Now we assume that $\operatorname{supp}(A)$ contains no 3-element sets, but it contains two disjoint 2 -element sets $\{i, j\}$ and $\{k, l\}$. In this case, $\operatorname{supp}(B)$ contains the empty set and all singletons plus one of the following families of 2 -element sets, up to a suitable symmetry on the indices $i, j, k$, and $l$ :

1. $\emptyset$, 
2. $\{\{i, k\}\}$,

3. $\{\{i, k\},\{i, l\}\}$,

4. $\{\{i, k\},\{j, l\}\}$,

5. $\{\{i, k\},\{i, l\},\{j, l\}\}$,

6. $\{\{i, k\},\{i, l\},\{j, k\},\{j, l\}\}$.

Note that in subcase $1, A$ and $B$ are balls, and subcase 6 is identical with subcase 4 with the roles of $A$ and $B$ reversed. We use Lemma 9 and numeric comparisons to rule out the remaining cases.

Consider the monotone ball $C$ of radius 1 in the set of coordinates $[n] \backslash$ $\{i\}$. This is an $r$-intersecting family. In subcases 2 and $3, i$ dominates all other coordinates, so we may assume that $p_{i}$ is maximal among all the $p_{m}(m \in[n])$. In both subcases considered, we have $2|C| \geq|A|+|B|$. In subcase 2 , this follows from $p_{i} \geq p_{k}$, while in subcase 3 , we again need to apply the inequality in Lemma 9 . In both subcases, we have $|A|>|B|$. Thus, $|A| \cdot|B|<|C|^{2}$, contradicting the maximality of the pair $(A, B)$.

A similar argument works in subcases 4 and 5 . Here $i$ does not dominate $l$ (nor does it dominate $j$, in subcase 4 ), but it dominates all other indices, and we can still assume by symmetry that $p_{i}$ is maximal. This implies that $|A|+|B|<2|C|$, so that $|A| \cdot|B|<|C|^{2}$, a contradiction.

Case 3. Finally, assume that Cases 1 and 2 do not hold. In this case, for any pair $T, U \in \operatorname{supp}(A)$, we have $|T \cup U| \leq 3$ and, hence, $\operatorname{supp}(B) \supseteq \operatorname{supp}(A)$. We can further assume by symmetry that $\operatorname{supp}(B)$ contains no 3 -element set and no pair of disjoint 2-element sets. This implies $\operatorname{supp}(A)=\operatorname{supp}(B)$ so that $A=B$. In this case, $\operatorname{supp}(A)$ contains the empty set, the singletons, and a containmentmaximal intersecting family of pairs. There are only two types of such families to consider:

1. (star) $\operatorname{supp}(A)$ contains the empty set, all singletons, and all pairs containing some fixed coordinate $i \in[n]$.

2. (triangle) $\operatorname{supp}(A)$ contains the empty set, all singletons, and the pairs formed by two of the three distinct coordinates $i, j, k \in[n]$.

Here subcase 1 is not possible, as $i$ is not a relevant coordinate for $A$. In subcase 2, we may once again assume that $p_{i}$ is maximal. We use the same $r$-intersecting family $C$ as in Case 2. To see that $|A|=|B|<|C|$ (a contradiction), we use Lemma 9.

To extend Theorem 8 to somewhat larger values of relevant coordinates (that is, to verify Conjecture 3, for instance, for the case where there are $r+4$ relevant coordinates), we would have to go through a similar case analysis as above. We would have to consider much more cases that correspond to containmentmaximal pairs of set systems $(U, V)$ with $|u \cup v|$ bounded for $u \in U$ and $v \in V$. This seems to be doable, but the number of cases to consider grows fast.

Now we can prove our main theorem, verifying Conjecture 3 in several special cases. 
Proof of Theorem $\%$. The statement about the case $p_{1}>r+1$ readily follows from Lemma 2, as in this case the condition

$$
\frac{2}{p_{r+1}}+\sum_{i=1}^{r} \frac{1}{p_{i}} \leq 1
$$

holds.

To prove the other two statements in the theorem, we assume that $A$ and $B$ form a maximal $r$-cross-intersecting pair. We also assume without loss of generality that all coordinates are relevant for both families (simply drop the irrelevant coordinates).

By Theorem 6, we have $\prod_{i=1}^{r} p_{i} \geq \prod_{i=1}^{n}\left(p_{i}-1\right)^{1-2 / p_{i}}$, and thus

$$
\prod_{i=1}^{r} \frac{p_{i}}{\left(p_{i}-1\right)^{1-2 / p_{i}}} \geq \prod_{i=r+1}^{n}\left(p_{i}-1\right)^{1-2 / p_{i}} .
$$

Here the function $x /(x-1)^{1-2 / x}$ is decreasing for $x \geq 3$, while $(x-1)^{1-2 / x}$ is increasing, and we have $p_{i} \geq p_{1} \geq 3$. Therefore, we also have

$$
\begin{gathered}
\prod_{i=1}^{r} \frac{p_{1}}{\left(p_{1}-1\right)^{1-2 / p_{1}}} \geq \prod_{i=r+1}^{n}\left(p_{1}-1\right)^{1-2 / p_{1}}, \\
p_{1}^{r} \geq\left(p_{1}-1\right)^{n\left(1-2 / p_{1}\right)} \\
n \leq \frac{r \log p_{1}}{\left(1-2 / p_{1}\right) \log \left(p_{1}-1\right)} .
\end{gathered}
$$

Simple calculation shows that the right-hand side of the last inequality is strictly smaller than $r+4$ if $p_{1} \leq t(r)$ for some function $t(r)=r / 2+o(r)$ and, in particular, for $p_{1}=r+1 \geq 5$. In this case, we have $n \leq r+3$ relevant coordinates. Thus, Theorem 8 applies, yielding that $A$ and $B$ are balls. This proves the last statement of Theorem 7 .

For the proof of the second statement, note that we have already established that $A$ and $B$ are balls in up to $r+3$ coordinates. Theorem 4 tells us that the pair of radii must be $(0,0),(0,1),(1,1)$, or $(1,2)$. Simple calculation shows that the first possibility (fixing the smallest $r$ coordinates) is always optimal, and the cases where the two radii are unequal never yield maximal $r$-cross-intersecting pairs. Finally, the construction with a ball of radius 1 in $r+2$ coordinates matches the family obtained by fixing the $r$ smallest coordinates if and only if all relevant coordinates satisfy $p_{i}=r+1$. This completes the proof of Theorem 7 .

\section{Coordinates with $p_{i}=2$}

In many of our results, we had to assume $p_{i}>2$ for all coordinates of the size vector. Here we elaborate on why the coordinates $p_{i}=2$ behave differently. 
For the simple characterization of the cases of equality in Theorem 1, the assumption $k \neq 2$ is necessary. Here we characterize all maximal cross-intersecting pairs in the case $k=2$.

Let $p=\left(p_{1}, \ldots, p_{n}\right)$ be a size vector of positive integers with $k=\min _{i} p_{i}=2$ and let $I=\left\{i \in[n]: p_{i}=2\right\}$. For any set $W$ of functions $I \rightarrow[2]$, define the families

$$
\begin{aligned}
& A_{W}=\left\{x \in S_{p}: \exists f \in W \text { such that } x_{i}=f(i) \text { for every } i \in I\right\}, \\
& B_{W}=\left\{y \in S_{p}: \nexists f \in W \text { such that } y_{i} \neq f(i) \text { for every } i \in I\right\} .
\end{aligned}
$$

The families $A_{W}$ and $B_{W}$ are cross-intersecting for any $W$. Moreover, if $|W|=$ $2^{|I|-1}$, we have $\left|A_{W}\right| \cdot\left|B_{W}\right|=\left|S_{p}\right|^{2} / 4$, so they form a maximal cross-intersecting pair. Note that these include more examples than just the pairs of families described in Theorem 1, provided that $|I|>1$.

We claim that all maximal cross-intersecting pairs are of the form constructed above. To see this, consider a maximal pair $A, B \subseteq S_{p}$. We know from the proof of Theorem 1 that $x \in A$ if and only if $x^{\prime} \notin B$, where $x^{\prime}$ is defined by $x_{i}^{\prime}=\left(x_{i}+1 \bmod p_{i}\right)$ for all $i \in[n]$. Let $j \in[n]$ be a coordinate with $p_{j}>2$. By the same argument, we also have that $x \in A$ holds if and only if $x^{\prime \prime} \notin B$, where $x_{i}^{\prime \prime}=x_{i}^{\prime}$ for $i \in[n] \backslash\{j\}$ and $x_{j}^{\prime \prime}=\left(x_{j}+2 \bmod p_{j}\right)$. Thus, both $x^{\prime}$ and $x^{\prime \prime}$ belong to $B$ or neither of them does. This holds for every vector $x^{\prime}$, implying that $j$ is irrelevant for the family $B$ and thus also for $A$.

As there are no relevant coordinates for $A$ and $B$ outside the set $I$ of coordinates with $p_{i}=2$, we can choose a set $W$ of functions from $I$ to [2] such that $A=A_{W}$. This makes

$$
B=\left\{y \in S_{p}: y \text { intersects all } x \in A\right\}=B_{W} .
$$

We have $|A|+|B|=\left|S_{p}\right|$ and $|A| \cdot|B|=\left|S_{p}\right|^{2} / 4$ if and only if $|W|=2^{|I|-1}$.

The size vector $p=(2, \ldots, 2)$ of length $n$ is well studied. In this case, $S_{p}$ is the $n$-dimensional hypercube. If $r>1$, then all maximal $r$-cross-intersecting pairs have an unbounded number of relevant coordinates, as a function of $n$. Indeed, the density $|A| \cdot|B| /\left|S_{p}\right|^{2}$ is at most $1 / 4$ for cross-intersecting pairs $A, B \subseteq S_{p}$, and strictly less than $1 / 4$ for $r$-cross-intersecting families if $r>1$. Furthermore, if the number of relevant coordinates is bounded, then this density is bounded away from $1 / 4$, while if $A=B$ is the ball of radius $(n-r) / 2$ in all the coordinates, then the same density approaches $1 / 4$.

One can also find many maximal 2-cross-intersecting pairs that are not balls. For example, in the 3 -dimensional hypercube the families $A=\{0,0,0),(0,1,1)\}$ and $B=\{(0,0,1),(0,1,0)\}$ form a maximal 2-cross-intersecting pair.

Finally, we mention that there is a simple connection between the problem discussed in this paper and a question related to communication complexity. Consider the following two-person communication game: Alice and Bob each receive a vector from $S_{p}$, and they have to decide whether the vectors are $r$ intersecting. In the communication matrix of such a game, the rows are indexed by the possible inputs of Alice, the columns by the possible inputs of Bob, and an 
entry of the matrix is 1 or 0 corresponding to the "yes" or "no" output the players have to compute for the corresponding inputs. In the study of communication games, the submatrices of this matrix in which all entries are equal play a special role. The largest area of an all-1 submatrix is the maximal value of $|A| \cdot|B|$ for $r$-cross-intersecting families $A, B \subseteq S_{p}$.

Acknowledgment. We are indebted to G. O. H. Katona, R. Radoičić, and D. Scheder for their valuable remarks, and to an anonymous referee for calling our attention to the manuscript of Borg 5 .

\section{References}

1. R. Ahlswede and L. H. Khachatrian, The diametric theorem in Hamming spacesoptimal anticodes, Adv. in Appl. Math., 20, 429-449 (1998).

2. C. Berge, Nombres de coloration de l'hypegraphe h-parti complet, Hypergraph Seminar, Lecture Notes in Math., Springer-Verlag, Heidelberg, 411, 13-20 (1974).

3. B. Bollobás, Combinatorics. Set Systems, Hypergraphs, Families of Vectors and Combinatorial Probability, Cambridge University Press, Cambridge (1986).

4. P. Borg, Intersecting and cross-intersecting families of labeled sets, Electron. J. Combin., 15 N9 (2008).

5. P. Borg, Cross-intersecting integer sequences, preprint, arXiv:1212.6955

6. M. Deza and P. Frankl, Erdős-Ko-Rado theorem-22 years later, SIAM J. Alg. Disc. Methods, 4, 419-431 (1983).

7. P. Erdös, C. Ko, and R. Rado, Intersection theorems for systems of finite sets, Quart. J. Math. Oxford Ser. 2, 12, 313-318 (1961).

8. P. Frankl, Extremal set systems, in Handbook of Combinatorics (R. Graham et al., eds.), Elsevier, Amsterdam, 1293-1329 (1995).

9. P. Frankl and Z. Füredi, The Erdős-Ko-Rado theorem for integer sequences, SIAM J. Algebraic Discrete Methods, 1, 376-381 (1980).

10. P. Frankl, S. J. Lee, M. Siggers, and N. Tokushige, An Erdős-Ko-Rado theorem for cross-intersecting families, J. Combin. Theory Ser. A, 128, 207-249 (2014).

11. P. Frankl and N. Tokushige, The Erdős-Ko-Rado theorem for integer sequences, Combinatorica, 19, 55-63 (1999).

12. C. Greene and D. J. Kleitman, Proof techniques in the ordered sets, in: Studies in Combinatorics, Math. Assn. America, Washington DC, 22-79 (1978).

13. A. J. W. Hilton, An intersection theorem for a collection of families of subsets of a finite set, J. London Math. Soc., 2, 369-384 (1977).

14. G. O. H. Katona, A simple proof of the Erdős-Ko-Rado theorem, J. Combin. Theory Ser. B, 13, 183-184 (1972).

15. M. L. Livingston, An ordered version of the Erdös-Ko-Rado theorem, J. Combin. Theory Ser. A, 26, 162-165 (1979).

16. A. Moon, An analogue of the Erdős-Ko-Rado theorem for the Hamming schemes H(n,q), J. Combin. Theory Ser. A, 32, 386-390 (1982).

17. L. Pyber, A new generalization of the Erdős-Rado-Ko theorem, J. Combin. Theory Ser. A, 43, 85-90 (1986).

18. D. Stanton, Some Erdős-Ko-Rado theorems for Chevalley groups, SIAM J. Algebraic Discrete Methods 1, 160-163 (1980).

19. N. Tokushige, Cross $t$-intersecting integer sequences from weighted Erdős-Ko-Rado, Combin. Prob. Comput., 22, 622-637 (2013). 
20. H. Zhang, Cross-intersecting families of labeled sets, Electron. J. Combin. 20(1), P17 (2013). 